\title{
116 años de la Revista Chilena de Historia Natural: Breve relato de dos antes y un después
}

\section{6 years of Revista Chilena de Historia Natural: A brief story of two befores and an after}

\author{
PATRICIO A. CAMUS \\ Editor Jefe \\ Revista Chilena de Historia Natural \\ Departamento de Ecología, Facultad de Ciencias, Universidad Católica de la Santísima Concepción, \\ Casilla 297, Concepción, Chile \\ e-mail: pcamus@ucsc.cl
}

\begin{abstract}
RESUMEN
El año 2013 marcará el cierre de una etapa importante para la Revista Chilena de Historia Natural (RCHN), ya que a partir de 2014 comenzará a publicarse a través de la plataforma SpringerOpen, incluyendo algunos cambios relevantes en su diseño, manejo editorial, e idioma, entre otros. Por lo tanto en este artículo sintetizo algunos aspectos clave en la evolución de RCHN desde su fundación a fines del siglo 19, a través de dos períodos históricos: (a) 1897-1963, donde destaca la figura de su creador Carlos E. Porter y el rol de la Sociedad Chilena de Historia Natural, y (b) 1983-2013, su etapa reciente ligada a la Sociedad de Biología de Chile. Adicionalmente presento un breve análisis de la situación y el impacto de RCHN en su etapa moderna, incluyendo información inédita que muestra la preferencia (vía citación) por distintos aspectos de sus contenidos (categoría de artículo, área disciplinaria, tipo de ambiente), y un ranking de los artículos más citados vs. aquellos más consultados online, que revela dos caras distintas del impacto de la revista.
\end{abstract}

Palabras clave: Chile, historia natural, impacto.

\section{ABSTRACT}

The year 2013 will mark the end of an important period for Revista Chilena de Historia Natural (RCHN), as from 2014 it will start to be published through the SpringerOpen platform, including some conspicuous changes in design, editorial management and language, among others. Therefore in this article I summarize some key aspects in the evolution of RCHN since its foundation in the late 19th century, through two historical periods: (a) 1897-1963, which highlights the figure of its creator Carlos E. Porter and the role of the Sociedad Chilena de Historia Natural (Chilean Society of Natural History), and (b) 1983-2013, its recent period linked to the Sociedad de Biología de Chile (Biology Society of Chile). Additionally, I present a brief analysis of the situation and the impact of RCHN in its modern period, including unpublished information showing the preference (via citation) for different aspects of its contents (article category, disciplinary area, environment type), and a ranking of the most cited articles vs. those most consulted online, which reveals two different faces of the journal's impact.

Key words: Chile, impact, natural history.

\section{INTRODUCCIÓN}

A partir de Enero 2014 la Revista Chilena de Historia Natural (RCHN) iniciará otra etapa en su larga trayectoria, ya que comenzará a publicarse en la plataforma SpringerOpen, en función de un acuerdo suscrito con la editorial Springer por la Sociedad de Biología de Chile (SBC), propietaria de la revista. En su nueva etapa RCHN tendrá un nuevo editor (el Dr. F. Patricio Ojeda) y seguirá bajo la tuición de la SBC, si bien mostrará cambios importantes en su diseño gráfico y portada, su modalidad de publicación (electrónica, con procesos completamente online), la estructura y funciones de su cuerpo editorial, y su idioma (inglés únicamente), entre otros aspectos, que 
en la práctica configuran una nueva versión de la revista.

En efecto este número final de 2013 (Vol. 864) concluye una etapa significativa de RCHN, y como actual Editor creí pertinente sintetizarla en este artículo, aunque con cierta parcialidad debido a un largo vínculo como lector, revisor y autor de la revista, donde -por circunstancias fortuitas- desde 1995 serví como Editor de Producción, Ed. Asociado y Ed. Jefe (funciones que ahora cambiarán, por lo que curiosamente tendré el anecdótico honor de haber sido el único que pasó por sus tres cargos editoriales). Pero ciertamente estoy muy lejos de ser el único o el de mayor antigüedad en forjar nexos con RCHN, como atestigua por ejemplo la labor del Dr. Julio Gutiérrez (Chile) y el Dr. Peter Feinsinger (USA), que han servido por $25 \mathrm{y}$ más de 23 años respectivamente como Editores Asociados. Más aún, al considerar la percepción de quienes han sido lectores, autores, revisores y editores de RCHN a través del tiempo, es posible apreciar cómo el gentilicio "chilena" ha ido adquiriendo una connotación cada vez más neutra, simplemente como un término más de su nombre. Esto refleja que la RCHN actual es sin duda más regional que "chilena" (más adelante se verá por qué ha retenido este nombre), y de hecho si ha podido mantenerse y prosperar ha sido en gran medida por recibir una colaboración internacional amplia y generosa. En su nueva etapa, sin embargo, RCHN intentará emerger en el complejo mercado mundial de la publicación científica (y en el contexto de un cambio global en las condiciones de producción del conocimiento), marcado por una fuerte competencia y una guerra desatada por el rating. El tiempo dirá si las cartas fueron bien jugadas en ese contexto, y la evaluación de ese futuro -que corresponde al "después" mencionado en el título de este artículo- quedará en manos de la SBC y el nuevo editor, a quienes por cierto deseo todo el éxito posible.

En este contexto, como editor saliente consideré apropiado dejar una imagen de lo que ha sido RCHN hasta ahora, y como es obvio preferí hacerlo en español ya que quizá no habrá otra oportunidad en el futuro. Primero resumiré algunos momentos clave en el recorrido de $\mathrm{RCHN}$, refiriéndome a los dos "antes" indicados en el título que corresponden a dos etapas históricas muy distintas y separadas por una discontinuidad de casi 20 años, y que sin embargo lograron articularse en una misma trayectoria. En esta parte incluyo datos de una recopilación de documentos en su mayoría inéditos realizada por el ex Editor de RCHN, Dr. Ernst R. Hajek Girardi (a quien mencionaré en toda justicia más adelante), y que espero puedan ser conocidos en su totalidad en el futuro. Luego resumiré brevemente la situación y el impacto de RCHN en su etapa más reciente, incluyendo algunos datos que pueden ser de especial interés para lectores y autores ya que no han sido presentados o analizados anteriormente, $\mathrm{y}$ que revelan distintas caras de la revista.

\section{LOS DOS “ANTES” DE RCHN}

\section{Primera etapa: fundación, auge, y caída}

En lo que respecta a la fundación de RCHN, la fecha que ha quedado inscrita en la tradición y los registros formales -por razones más prácticas que de rigor histórico- es el 4 de Octubre de 1897, el día en que se publicó su primer número. Su creador fue Carlos Emilio Porter Mosso (1867-1942), un joven profesor chileno de la ciudad de Valparaíso, cuya intención explícita fue impulsar la investigación chilena en el ámbito de la historia natural en sentido amplio, y así lo reflejó en el nombre de su revista. 116 años después, no cabe duda de que el objetivo de Porter se ha cumplido con creces, aunque al momento de fundar RCHN probablemente no imaginó que alcanzaría prestigio internacional y llegaría a ser una de las publicaciones científicas más antiguas en Hispanoamérica y el mundo.

Porter no solo fundó RCHN, sino que prácticamente la convirtió en una cruzada personal y la dirigió en forma incansable durante 44 años, casi literalmente hasta el fin de su vida. El enfoque innovador, visionario y riguroso de Porter logró que RCHN fuera reconocida en otros países, recibiendo varias distinciones internacionales y atrayendo a importantes investigadores y ganadores del Nobel a publicar trabajos en ella. Pero más allá de su íntimo nexo con RCHN, Porter fue de hecho un figura central en el desarrollo, institucionalización, descentralización e internacionalización de la ciencia naturalista chilena entre los siglos 19 y 20 , a tal punto 
que su obra personal constituye por sí sola un período histórico brillante de la ciencia en Chile (e.g., véase Courard 1968), con RCHN como su mejor exponente (Bahamonde 1983).

La energía, capacidad, creatividad y emprendimiento desplegados por Porter fueron extraordinarios para un científico de su época, y sus acciones y logros resultan envidiables para cualquier científico actual, ya que por ejemplo: (a) publicó más de 400 artículos y libros (productividad que pocos alcanzan hoy en día); (b) fue miembro de 69 sociedades científicas de Chile, Latinoamérica, Europa y EE.UU.; (c) colaboró en 21 revistas científicas chilenas y extranjeras; (d) fundó y dirigió RCHN, el "Boletín del Museo de Historia Natural de Valparaíso", y los "Anales de Zoología Aplicada”; (e) fundó y/o dirigió 6 sociedades científicas chilenas y extranjeras, y 5 institutos, laboratorios y museos en distintos lugares de Chile; y (f) fue profesor y catedrático honorario en varias instituciones chilenas y extranjeras, recibiendo 18 premios y distinciones en Argentina, Brasil, EE.UU., España, Francia, Inglaterra, Perú y Venezuela, y Doctorados Honoris Causa en Francia y EE.UU. En este contexto, Porter fue sin duda un gran impulsor y embajador de la ciencia chilena, aunque su lugar en la historia apenas está comenzando a ser reconocido apropiadamente (para más antecedentes biográficos sobre Porter véase Bahamonde 1983, Etcheverry 1988, y un excelente compendio en Jaksic et al. 2012, pp. 127-138).

Hacia el primer tercio del siglo 20, $\mathrm{RCHN}$ ya era una revista consolidada y de prestigio cuando su destino se unió al de la naciente Sociedad Chilena de Historia Natural (SCHN), de la que Porter fue uno de sus fundadores y además su director. De hecho, aun cuando la SCHN se constituyó en una reunión efectuada el 26 de Julio de 1926, sus socios propusieron que la fecha oficial de su fundación fuera el 4 de Octubre, en honor del aniversario número 30 de RCHN, la cual en adelante sería auspiciada oficialmente por la $\mathrm{SCHN}$, quien además sería su propietaria desde 1941. En todo este período, Porter, más que un director, fue realmente el motor de RCHN, y trabajó activamente para conseguir los auspicios y fondos que permitieran mantenerla viva. Tras la muerte de Porter, sin embargo, su ausencia se hizo sentir, y la revista comenzó a enfrentar diversos problemas y el desinterés de instituciones que antes la apoyaban. No obstante RCHN siguió publicándose en virtud de los notables y prolongados esfuerzos de varias personas y organizaciones, incluyendo por cierto a quienes sucedieron a Porter en su dirección: Francisco Riveros Zúñiga en el período 1941-51, Nibaldo Bahamonde Navarro en 1952-55, y Ernst Hajek en 1956-63.

El final de esta etapa de RCHN fue más bien dramático, y según comenta Hajek (documentos no publicados), marcado por dificultades financieras (e.g., fondos comprometidos entregados tardíamente o nunca, subvenciones aprobadas que no se reajustaban o eran suspendidas), y numerosas gestiones ante organismos privados y públicos (e.g., universidades, empresas y CONICYT). Pese a los esfuerzos, sin embargo, la SCHN finalmente se vio superada por las dificultades, y en 1963 RCHN simplemente cesó de publicarse.

\section{Segunda etapa: renacimiento y auge}

Si bien la ausencia de RCHN se prolongó hasta 1982, no podría calificarse como una "desaparición" ya que la presencia y la memoria de la revista, siempre ligadas a la figura de Porter, se mantuvieron latentes en la comunidad naturalista. En tal sentido, la expresión más apropiada sería -como indicó Jaksic (1997)- que RCHN estuvo "durmiente", a la espera de una oportunidad. En paralelo, sin embargo, el quehacer biológico también había evolucionado, dando cabida a las disciplinas ecológicas que se encontraban en fuerte desarrollo (véase Jaksic et al. 2012), pero los naturalistas contemporáneos ya no contaban con una revista científica acorde a sus necesidades.

En este nuevo escenario, la oportunidad para RCHN surgió en 1981 precisamente a través de su último director, y miembro de la SCHN, el Dr. Ernst Hajek, que en ese momento también era Presidente de la SBC. Buscando canalizar la inquietud de los miembros naturalistas de la SBC, Hajek consideró que la solución más apropiada no era crear una revista nueva sino reactivar RCHN, e inició negociaciones proponiendo el traspaso legal de la revista desde la SCHN a la SBC, iniciativa que encontró excelente 
recepción en la primera, ante la posibilidad de dar continuidad a la revista en manos de una sociedad con alta convocatoria. Así, el traspaso a la SBC se formalizó en 1982, incluyendo algunas condiciones solicitadas por la SCHN que, entre otras, incluían mantener el nombre de la revista, continuar su numeración original, y elegir a su director de común acuerdo. En este punto es pertinente notar que además de la condición requerida por la SCHN, y como parte de los trámites legales, el nombre "Revista Chilena de Historia Natural" quedó inscrito como marca en los registros de propiedad intelectual del Gobierno de Chile, con lo cual en los años posteriores resultaría casi impracticable considerar ya sea un cambio de nombre o su eventual traducción al inglés (notando que estas opciones han sido sugeridas y evaluadas más de una vez).

De esta forma, RCHN fue reactivada bajo la tuición de la SBC, donde inició su etapa moderna en manos de Ernst Hajek, quien fue designado como candidato natural para conducir la revista, y que en consonancia con la figura de Porter, ha sido además uno de los grandes impulsores de la ecología y las ciencias ambientales en Chile. En 1983, RCHN reanudó su publicación con el $\mathrm{N}^{\circ} 1$ del Volumen 56, incluyendo una breve editorial donde Hajek señalaba que "Al adecuar la actual revista a la realidad científica del presente, los editores creen estar preservando el espíritu innovador de su fundador", una frase que a mi juicio materializa el sentido y la continuidad de RCHN tanto en el tiempo como en la percepción de los naturalistas. De hecho esta reedición de RCHN preservó el espíritu y muchos elementos de la publicación original, al punto que sus casi 20 años de ausencia parecen solo una anécdota.

Al reaparecer RCHN, Hajek tomó la pronta iniciativa de someterla al "Institute for Scientific Information” (ISI, hoy reemplazado por Thomson Reuters) para que fuese incluida en la importante base de datos del CC/ABES ("Current Contents /Agriculture, Biology \& Environmental Sciences”). Sin embargo ISI no se dejó seducir por la larga historia previa de RCHN, y su respuesta llegada el 14 de Septiembre de 1983 fue negativa, pero dando opción de reconsiderar la revista en unos dos años más, y según se indicaba perspicazmente, "después que haya tenido la oportunidad de reestablecerse a sí misma en la comunidad científica”. Con la conducción de Hajek, visionaria al igual que la de Porter, RCHN retomó rápidamente su regularidad y estándares y el 22 de Agosto de 1985 fue aceptada en el grupo mundial de revistas ISI, lo que se concretó con la aparición de su Vol. $58 \mathrm{~N}^{\circ} 2$ en la edición de CC/ABES del 10 de febrero de 1986.

El resto de la historia es más conocido, y todo lo hecho por RCHN está disponible en bases de datos internacionales tanto públicas como privadas, por lo que solo agregaré dos curiosidades para malgastar el tiempo del lector. Una, tan interesante como inútil, es que Porter fundó RCHN a los 30 años, la SCHN se fundó a los 30 años de RCHN, y la segunda etapa de RCHN re-fundada duró 30 años en que se publicaron 30 volúmenes, y sería deseable que RCHN honrara esta coincidencia durando al menos otros 30 años. La otra es el bajo número de editores que ha tenido RCHN, como destacó Jaksic (1997) en su editorial por los 100 años de RCHN notando que hubo apenas 5 en un siglo, aunque hasta hoy ya suman 8 : Carlos E. Porter (1897-1941), Francisco Riveros (1942-51), Nibaldo Bahamonde (1952-55), Ernst R. Hajek (1956-63, 1983-91), Fabián M. Jaksic (1992-98), F. Patricio Ojeda (1999-2002), Luis A. Ebensperger (2003-08), y quien escribe (200913). Incluso al descontar el receso de RCHN serían solo 8 editores en 97 años de publicación efectiva, e interesantemente este número por ahora no aumentará ya que el próximo editor (F.P. Ojeda) es también un ex editor.

\section{LA SITUACIÓN DE RCHN EN SU ETAPA MODERNA} (1983-2013)

Esta sección presenta algunos datos de interés sobre el rendimiento de RCHN en distintos contextos, aunque en general me enfoco principalmente en aspectos internos de la revista para resaltar algunas de sus características. Para ello uso información de distintas fuentes, y conjuntos de datos que fueron revisados en distintos momentos y que no necesariamente abarcan los mismos períodos de tiempo. Dado que este artículo se enfoca en la historia de RCHN, consideré poco pertinente realizar comparaciones bibliométricas específicas con otras revistas, y dejaré ese ejercicio a los lectores interesados 
(si bien indico algunos indicios generales sin ánimo de apuntar a revistas particulares).

\section{Visibilidad e impacto de RCHN}

Hasta 2011, y considerando todas las áreas disciplinarias, en Latinoamérica existían 283 revistas ISI provenientes de 12 países, y me atrevería a decir que todas o la mayoría de ellas intentando mejorar o mantener sus rankings (o al menos no decaer significativamente), y compitiendo por captar autores dentro de sus áreas particulares. En este sentido RCHN fue una de la primeras revistas de Chile y Latinoamérica en ingresar a ISI, y de acuerdo al Factor de Impacto de Thomson Reuters, entre 1990 y 2004 estuvo entre las 12 mejores considerando todas las áreas (Luna-Morales \& Collazo-Reyes 2007), lo cual por un buen tiempo le permitió prosperar manteniendo una posición de relativo privilegio y prestigio entre sus pares del área naturalista, pese a ser reconocida como una revista exigente (y de hecho lo es: su tasa promedio de rechazo en los últimos 10 años ha sido $\sim 55 \%$, con máximos de hasta $70 \%$ en ciertos momentos).

A fines de la década 2010, sin embargo, la ampliación online de la base de datos ISI (“Science Citation Index Expanded ${ }^{\mathrm{TM}}$ "), con $\sim 4000$ títulos adicionales incluyendo varias revistas con tradición en Latinoamérica y Chile, intensificó la competencia para $\mathrm{RCHN}$ que en ese momento además era comparada con mayor frecuencia con revistas extranjeras (principalmente de EE.UU. y Europa) contra las que era a su vez menos competitiva. Este hecho fue una suerte de alarma para una RCHN que evidenciaba cierta tendencia a "dormir en sus laureles", mientras su Factor de Impacto -pese a tener una clara tendencia positiva- no mostraba aumentos significativos, configurando un escenario más bien adverso, en particular frente a sus pares internacionales, debido a una relación impacto/rechazo relativamente baja. Por ello en los últimos 5 años el esfuerzo se centró en mejorar la difusión y visibilidad de RCHN, y en potenciar la calidad, efectividad y disponibilidad de contenidos online de su sitio web oficial (http://rchn.biologiachile. cl), que ya a fin de 2010 incluía un repositorio propio con todos sus artículos (en PDF) publicados desde 1897. En este contexto RCHN debe agradecer la respuesta positiva de sus lectores y contribuyentes, que se tradujo en un aumento visible en su impacto desde 2010 en adelante. Un síntoma de este crecimiento y mayor visibilidad son las visitas al sitio web oficial de $\mathrm{RCHN}$, sin duda la menor y menos importante de las fuentes oficiales que albergan o indexan la revista (e.g., Web of Knowledge, SciELO, SCImago y otras), pero que ya suma consultas de casi 90 países principalmente de las tres Américas, Europa y Asia (http://www3.clustrmaps. com/es/counter/maps.php?url=http://rchn. biologiachile.cl/; ClustrMaps $\left.{ }^{\circledR}\right)$.

La Fig. 1.A muestra la evolución del impacto de RCHN medida según: (a) el Factor de Impacto de Thomson Reuters (Journal of Citation Reports $\AA)$, donde en años recientes RCHN consolidó su posición como una de la revistas ISI con mejor desempeño entre sus pares de Chile e Hispanoamérica; y (b) el índice SJR de SCImago, que combina el número de citas recibidas por una revista y la
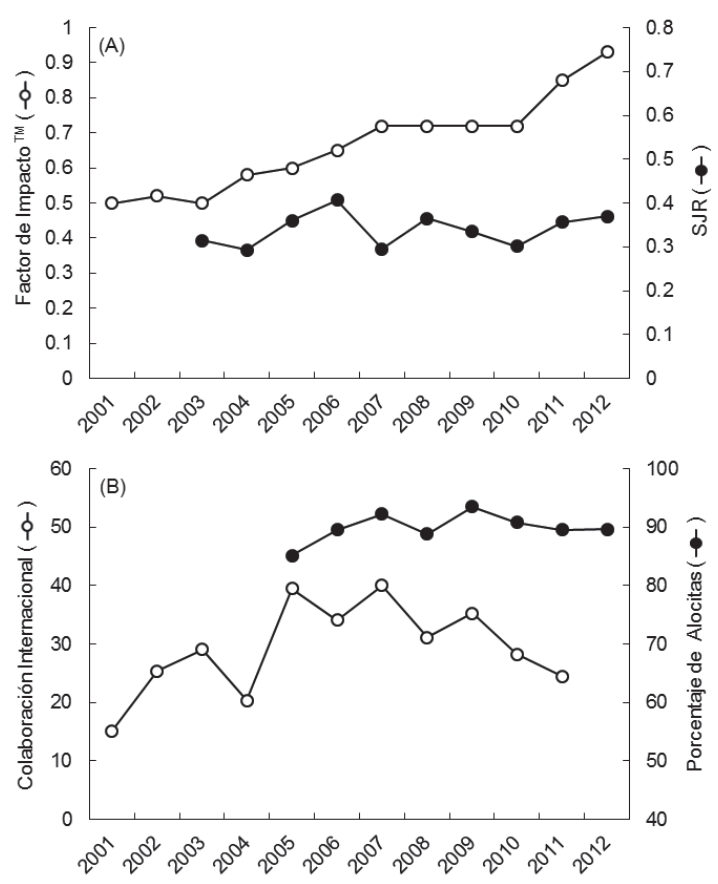

Fig. 1: Algunas estadísticas de la Revista Chilena de Historia Natural. (A) Dos índices de citación: Factor de impacto de Thomson Reuters e índice SJR de SCImago. (B) Colaboración internacional y porcentaje de alocitas.

Some statistics of Revista Chilena de Historia Natural. (A) Two citation indices: Thomson Reuters' Impact Factor and SCImago's SJR index. 
importancia o prestigio de aquellas revistas que la citaron (i.e. la "influencia" de un artículo promedio), donde RCHN ha mantenido un desempeño comparativamente alto con pocas fluctuaciones. Sin embargo, bastante antes de alcanzar su mayor impacto RCHN ya destacaba en la base de datos del "Directory of Open Access Journals" (DOAJ). En un análisis de Giglia (2010), considerando 4833 revistas incluidas en el DOAJ y datos del JCR de 2008, RCHN se ubicaba en la posición 96 por impacto (percentil 20) y la posición 8 por índice de inmediatez (percentil 1.7). Por cierto los índices de RCHN no eran comparables a los de revistas de alto impacto a nivel mundial, pero al considerar las 479 revistas incluidas tanto en ISI como en DOAJ, el mismo análisis mostró que entre aquellas 225 revistas con impacto comparativamente menor, RCHN alcanzaba la posición 1 por su rendimiento en impacto vs. inmediatez (Giglia 2010).

Los interesados en observar el desempeño de RCHN respecto al de otras revistas pueden recurrir entre otros recursos al portal "SCImago Journal \& Country Rank" (http:// www.scimagojr.com/), que permite comparar revistas desplegando diversos índices, algunos de los cuales son muy informativos o reveladores. La Fig. 1-B muestra por ejemplo el índice de colaboración internacional (\% de documentos citables con autores de más de un país), donde en relación a otras revistas biológicas de Chile y Latinoamérica, RCHN presenta valores comparativamente altos (y a veces muy altos), lo que sin duda aporta solidez a sus contenidos aunque no refleja con claridad el grado de internacionalización (e.g., no considera la diversidad de nacionalidades ni el caso frecuente de artículos de países distintos pero con autores de un mismo país). En este sentido, desde 2009 a 2013 RCHN publicó 232 artículos ( $52 \%$ de ellos en idioma inglés) evaluados por revisores de 25 países, y cuya autoría se distribuyó entre investigadores de 23 países, donde los 10 países con más autores en artículos distintos fueron (en orden descendente) Chile, Argentina, México, Brasil, Estados Unidos, España, Alemania, Nueva Zelanda, Australia y Colombia.

Otro punto relevante es la incidencia de las autocitas vs. alocitas, considerando que el Factor de Impacto de Thomson Reuters aumenta con la autocitación (no así el valor de otros índices). Este aspecto no siempre es considerado en los análisis comparativos de impacto, aunque herramientas como las que provee SCImago (op. cit.) permiten observar y comparar fácilmente el nivel de autocitas entre revistas, en ocasiones con alguna sorpresa. Después de todo vivimos en la era del "rating", donde la máxima "publish or perish" ya es anticuada (hoy parece ser "achieve high impact or perish"), al punto que Thomson Reuters ha suprimido del JCR un alto número de revistas por mostrar patrones de citación anómalos, a lo que se agrega el descubrimiento reciente de los "carteles de citación" (no incluyo referencias ya que en los medios hay abundante información sobre el tema). En este contexto debo destacar que RCHN no ha aplicado políticas de autocitación para favorecer su impacto, y sus autocitas solo reflejan la tendencia usual de los autores a citar algunos trabajos propios. De hecho la Fig. 1.B (con datos obtenidos de SCImago) muestra que RCHN ha mantenido un porcentaje de alocitas muy alto, por lo cual tiene la tranquilidad de que su Factor de Impacto -sea bajo o alto según con quien se comparees transparente y se debe esencialmente a un reconocimiento externo.

Más allá del "rating", sin embargo, a mi juicio hay dos razones principales por las cuales RCHN debe cuidar su impacto, que no se relacionan con la posibilidad de obtener éxito (o ganancias), aun si esto llega a ocurrir. Una es el simple hecho de que una revista científica es un vehículo para validar conocimiento, función que amerita un alto grado de rigurosidad y responsabilidad, y la otra es que si RCHN gana aceptación esto la vuelve un medio más eficaz para difundir el trabajo de nuestra comunidad científica.

\section{Otros impactos domésticos}

Porter fundó RCHN concibiéndola como una revista de espectro temático muy amplio, y este rasgo se ha mantenido en su etapa reciente, como constataba hace casi dos décadas una editorial que evaluó la representación de áreas disciplinarias en los artículos publicados desde 1983 a 1995 (Camus 1995), aspecto que luego fue resaltado por Jaksic (1997). Actualmente los énfasis de los autores (y en parte de la revista) son algo distintos en cuanto a la forma de definir las disciplinas, pero la 
diversidad temática genera mucho menos interés que su impacto. Por ello en este punto resumiré algunos datos (obtenidos de "The Web of Knowledge") derivados de un análisis efectuado en 2010 de la citación de los trabajos publicados por RCHN en el período 2004- 2009, vistos de diferentes perspectivas (el período fue escogido arbitrariamente con el propósito de tener una imagen del momento reciente de RCHN).

Al considerar los trabajos según el tipo de contribución, y enfocándose solo en los tres tipos más regulares y de contenido científico principalmente, el rendimiento de $\mathrm{RCHN}$ en términos del promedio $( \pm \mathrm{DE})$ del número de citas por artículo (cpa) fue el siguiente: (a) 242 Artículos de Investigación con $2.74 \pm 3.63$ cpa; (b) 10 Artículos de Revisión con $5.88 \pm$ 5.89 cpa; y (c) 17 Comentarios (una sección de ensayos críticos que hoy se denomina Forum) con $2.31 \pm 3.07$ cpa. Como suele ocurrir, las revisiones fueron mucho más citadas que los artículos regulares o los ensayos, aunque es interesante que estos últimos no tuvieran mayor diferencia. Curiosamente $\mathrm{RCHN}$ nunca ha tenido una política de privilegiar la publicación de revisiones, aunque es obvio que sería una buena estrategia para aumentar su citación, lo cual refleja que el impacto de la revista tampoco ha sido manejado abiertamente en este sentido, aunque en este caso habría sido algo más positivo.

Para tener una perspectiva disciplinaria, y considerando todos los tipos de contribución con contenidos científicos, los artículos fueron clasificados individualmente dentro de las 10 áreas definidas como ámbito temático oficial de RCHN. La Fig. 2.A muestra la citación promedio para cada área, la cual varió desde $3.48 \pm 5.38 \mathrm{cpa}$ (ecología de comunidades) hasta $1.71 \pm 2.37$ cpa (botánica), notando que el área botánica no incluyó trabajos en ecología vegetal (poblacional, comunitaria $\mathrm{u}$ otra). Un aspecto interesante es que la variación entre áreas fue relativamente baja, lo cual indica que a pesar de su amplio espectro temático, el impacto de RCHN no está dominado por una o pocas disciplinas particulares (i.e. que la probabilidad de citación de cualquier trabajo en cualquier área es relativamente similar).
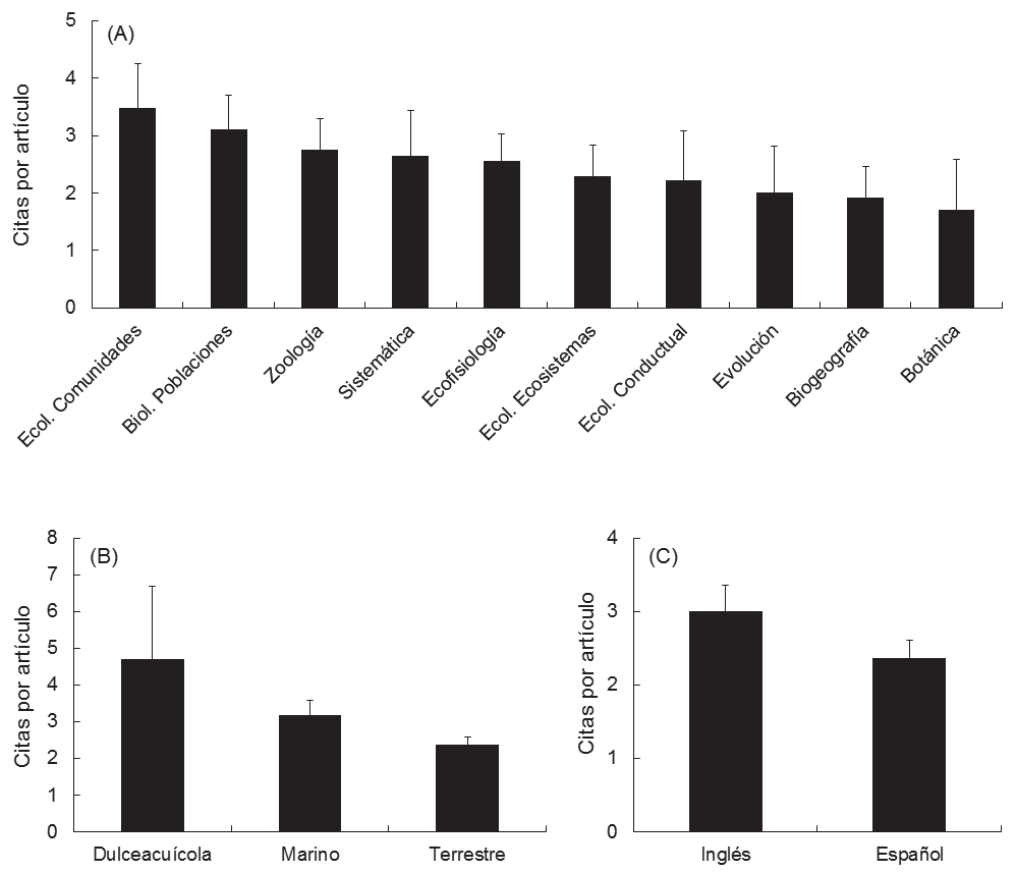

Fig. 2: Número promedio de citas por artículo en el período 2004-2009 (fuente: "The Web of Knowledge"), analizadas por (A) área disciplinaria, (B) tipo de ambiente, y (C) idioma de publicación.

Average number of cites per article in the period 2004-2009 (source: The Web of Knowledge), analyzed by (A) disciplinary area, (B) environment type, and (C) publication language. 
Por otra parte, la Fig. 2.B muestra la citación promedio pero según el tipo de ambiente estudiado, que varió desde $4.69 \pm$ 8.01 cpa (ambientes dulceacuícolas) hasta $2.36 \pm 2.95 \mathrm{cpa}$ (ambientes terrestres). Este resultado es doblemente paradójico (y sugerente) para la revista, ya que la citación es inversamente proporcional a la cantidad de artículos (162 terrestres, 64 marinos y solo 16 dulceacuícolas), y además porque tradicionalmente los contenidos de RCHN han estado dominados por la investigación en ambientes terrestres. Esto último sería también la causa de que los contenidos menos frecuentes sean a la vez más apreciados, $\mathrm{y}$ al menos en Chile es evidente que los ambientes de agua dulce han sido mucho menos investigados.

Por último la Fig. 2.C muestra la citación promedio según el idioma, revelando un valor algo mayor del inglés $(3.01 \pm 4.23 \mathrm{cpa})$ respecto al español $(2.36 \pm 2.49 \mathrm{cpa})$, aunque la diferencia a favor del inglés es mucho menor a la que podría haberse supuesto. Sin embargo (y esta es una hipótesis) este resultado podría estar asociado hasta cierto punto al hecho de que RCHN, como es usual en las revistas de nuestra región, ha tenido mayor difusión y acogida entre los investigadores hispanoparlantes, quienes suelen manejar ambos idiomas y probablemente no ejercían una fuerte discriminación por idioma al momento de citar un trabajo.

\section{Citación vs. consulta: las dos caras de RCHN}

Entre los resultados de nuestros análisis de citación hay uno en particular que fue un tanto inesperado, y revela que el "éxito" de los artículos publicados en RCHN debe ser evaluado desde distintas perspectivas. En este caso consideramos dos aspectos muy diferentes: (a) el número total de citas recibidas por los artículos publicados por RCHN hasta 2010 (con datos de "The Web of Knowledge"), que refleja básicamente su apreciación dentro de la comunidad científica; y (b) el número total de accesos a los artículos en el sitio web de RCHN en el portal SciELO ("Scientific Electronic Library Online”) desde Enero 2009 hasta Noviembre 2013, que incluye el acceso a los resúmenes y a las versiones HTML o PDF de los artículos, y que puede responder a objetivos e intereses muy diversos y difíciles de determinar a priori. Si bien las estadísticas disponibles en SciELO se limitan al rango de tiempo indicado (sin opción de incluir períodos previos), son suficientes (y elocuentes) como para permitir una comparación válida a nivel general.

La Tabla 1 muestra el ranking (en orden descendente) de los 20 artículos de RCHN más citados, aunque decidí omitir los números de citas para no desviar la atención hacia comparaciones puntuales. De estos artículos 10 son marinos, 8 terrestres y 2 de agua dulce, y sus temáticas son diversas aunque la gran mayoría abarca tópicos científicos amplios,

TABLA 1

Ranking de los 20 artículos más citados de la Revista Chilena de Historia Natural, basado en artículos publicados desde 1987 hasta 2010 y su número total de citas hasta 2010 (fuente: "The Web of Knowledge").

Ranking of the 20 most cited articles of Revista Chilena de Historia Natural, based on articles published from 1987 to 2010 and their total number of cites up to 2010 (source: The Web of Knowledge).

\begin{tabular}{|c|c|c|c|}
\hline Ranking & Autor(es) & Título & $\begin{array}{l}\text { Vol. }\left(\mathrm{N}^{\circ}\right) \\
\text { año }\end{array}$ \\
\hline 1 & $\begin{array}{l}\text { MARÍN V, RODRÍGUEZ L, } \\
\text { VALLEJO L et al. }\end{array}$ & $\begin{array}{c}\text { Efectos de la surgencia costera sobre la productividad } \\
\text { primaria primaveral de la bahía Mejillones del Sur } \\
\text { (Antofagasta, Chile) }\end{array}$ & 66(4) 1993 \\
\hline 2 & SHORT AD & $\begin{array}{l}\text { The role of wave height, period, slope, tide range and } \\
\text { embaymentisation in beach classifications: a review }\end{array}$ & $69(4) 1996$ \\
\hline 3 & HINOJOSA LF, VILLAGRÁN C & $\begin{array}{c}\text { Historia de los bosques del sur de Sudamérica, I: } \\
\text { antecedentes paleobotánicos, geológicos y climáticos del } \\
\text { Terciario del cono sur de América }\end{array}$ & $70(2) 1997$ \\
\hline
\end{tabular}




\begin{tabular}{|c|c|c|c|}
\hline Ranking & Autor(es) & Título & $\begin{array}{l}\text { Vol. }\left(\mathrm{N}^{\circ}\right) \\
\text { año }\end{array}$ \\
\hline 4 & $\begin{array}{l}\text { VILLAGRÁN C, HINOJOSA } \\
\text { LF }\end{array}$ & $\begin{array}{c}\text { Historia de los bosques del sur de Sudamérica, II: } \\
\text { Análisis fitogeográfico }\end{array}$ & 70(2) 1997 \\
\hline 5 & FEINSINGER P & $\begin{array}{c}\text { Approaches to nectarivore-plant interactions in the New } \\
\text { World }\end{array}$ & 60(2) 1987 \\
\hline 6 & CAMUS PA & Biogeografía marina de Chile continental & 74(3) 2001 \\
\hline 7 & $\begin{array}{l}\text { GONZÁLEZ HE, DANERI G, } \\
\text { FIGUEROA D et al. }\end{array}$ & $\begin{array}{c}\text { Producción primaria y su destino en la trama trófica } \\
\text { pelágica y océano profundo e intercambio océano- } \\
\text { atmósfera de } \mathrm{CO} 2 \text { en la zona norte de la Corriente de } \\
\text { Humboldt }\left(23^{\circ} \mathrm{S}\right) \text { : Posibles efectos del evento El Niño, } \\
\text { 1997-98 en Chile }\end{array}$ & 71(4) 1998 \\
\hline 8 & $\begin{array}{l}\text { LANCELLOTTI DA, } \\
\text { VASQUEZ JA }\end{array}$ & $\begin{array}{l}\text { Zoogeografía de macroinvertebrados bentónicos de la } \\
\text { costa de Chile: contribución para la conservación marina }\end{array}$ & $73(1) 2000$ \\
\hline 9 & $\begin{array}{l}\text { McLACHLAN A, DE RUYCK } \\
\text { A, HACKING N }\end{array}$ & $\begin{array}{l}\text { Community structure on Sandy beaches: patterns of } \\
\text { richness and zonation in relation to tide range and } \\
\text { latitude }\end{array}$ & 69(4) 1996 \\
\hline 10 & $\begin{array}{l}\text { BORZONE CA, SOUZA JRB, } \\
\text { SOARES AG }\end{array}$ & $\begin{array}{l}\text { Morphodynamic influence on the structure of inter and } \\
\text { subtidal macrofaunal communities of subtropical sandy } \\
\text { beaches }\end{array}$ & 69(4) 1996 \\
\hline 11 & CASTILLA JC, PAINE RT & $\begin{array}{l}\text { Predation and community organization on Eastern } \\
\text { pacific, temperate zone, rocky intertidal shores }\end{array}$ & $60(2) 1987$ \\
\hline 12 & BUCHER EH & Herbivory in arid and semi-arid regions of Argentina & $60(2) 1987$ \\
\hline 13 & $\begin{array}{l}\text { FERNANDEZ M, JARAMILLO } \\
\text { E, MARQUET PA et al. }\end{array}$ & $\begin{array}{c}\text { Diversidad, dinámica y biogeografía del ecosistema } \\
\text { costero bentónico de Chile: revisión y bases para } \\
\text { conservación marina }\end{array}$ & $73(4) 2000$ \\
\hline 14 & SMITH-RAMÍREZ C & $\begin{array}{l}\text { Los picaflores y su recurso floral en el bosque de la isla } \\
\text { de Chiloé, Chile }\end{array}$ & 66(1) 1993 \\
\hline 15 & DEFEO O & $\begin{array}{c}\text { Experimental management of an exploited sandy beach } \\
\text { bivalve population }\end{array}$ & $69(4) 1996$ \\
\hline 16 & $\begin{array}{l}\text { CAVIERES LA, PENAALOZA A, } \\
\text { ARROYO MTK }\end{array}$ & $\begin{array}{l}\text { Pisos altitudinales de vegetación en los Andes de Chile } \\
\text { central }\left(33^{\circ} \mathrm{S}\right)\end{array}$ & $73(2) 2000$ \\
\hline 17 & DUKES JS, MOONEY HA & $\begin{array}{c}\text { Disruption of ecosystem processes in western North } \\
\text { America by invasive species }\end{array}$ & $77(3) 2004$ \\
\hline 18 & BAYLY IAE & $\begin{array}{c}\text { Fusion of the genera Boeckella and Pseudoboeckella } \\
\text { (Copepoda) and revision of their species from South } \\
\text { America and sub-Antarctic islands }\end{array}$ & $65(1) 1992$ \\
\hline 19 & SOTO D, ZUÑIGA L & $\begin{array}{l}\text { Zooplankton assemblages of Chilean temperate lakes: A } \\
\text { comparison with North American counterparts }\end{array}$ & 64(3) 1991 \\
\hline 20 & $\begin{array}{l}\text { KENAG GJ, NESPOLO RF, } \\
\text { VÁSQUEZ RA et al. }\end{array}$ & $\begin{array}{c}\text { Limitaciones diarias y estacionales de tiempo y } \\
\text { temperatura sobre la actividad de degus }\end{array}$ & $75(3) 2002$ \\
\hline
\end{tabular}

que presumiblemente interesan a audiencias también amplias.

La Tabla 2 muestra el ranking (en orden descendente) de los 20 artículos más consultados, omitiendo los números de consultas por la misma razón anterior (pero los interesados pueden acceder al ranking completo en: http://scielolog.scielo.br//scielolog/scielolog. php? script $=$ sci_statart $\& \ln g=$ es $\&$ pid $=0716$ $078 \mathrm{X} \& \mathrm{app}=\mathrm{scielocl} \&$ server $=\mathrm{www}$. scielo. cl\&dti=20040101). En este caso hay 12 artículos terrestres, 3 marinos, 3 de agua dulce, y 2 ensayos conceptuales (donde el tipo de ambiente sería irrelevante). Sin embargo la mayoría de estos artículos es de naturaleza más bien aplicada o de interés para quienes abordan aspectos de tipo ambiental, aunque también en 
este contexto sus temas son diversos y tienden a abarcar tópicos amplios.

El contraste entre ambos rankings es evidente tanto en la naturaleza como en la composición de sus artículos (de hecho sólo 2 aparecen en ambas Tablas), y aunque las Tablas no lo muestran, estas diferencias tienden a mantenerse si se amplía el número de trabajos en ambos rankings. Más aún, los artículos de la Tabla 2 acumulan en conjunto cerca de 250000 consultas, un número totalmente inconmensurable con la citación en ámbitos científicos, pero varios de ellos acumulan un número bajo o muy bajo de citas en "The Web of Knowledge". Dado que no deseo perturbar a ningún autor cito como ejemplo dos artículos míos (publicados en 2002 y 2005, ambos relacionados con -o de interés para- el ámbito aplicado) que no están en la Tabla 2: uno se ubica en la posición 66 del ranking SciELO con 4934 consultas, y otro en la posición 72 con 4793 consultas, pero tienen solo 3 y 4 citas respectivamente en revistas ISI. Este patrón es bastante común entre los artículos del ranking SciELO, donde varios de los muy consultados pueden tener solo 1 o incluso 0 citas en ISI, y la conclusión obvia (pero no trivial) es que en general las consultas no se correlacionan con la citación, indicando además que los contenidos publicados por RCHN tienen usos y usuarios muy diferentes. De hecho hemos verificado que algunos artículos también acumulan visitas en SciELO porque son asignados como lectura en cursos universitarios de pre y postgrado (al menos en Chile esta es una práctica muy difundida, aunque la proporción

TABLA 2

Ranking de los 20 artículos más consultados de la Revista Chilena de Historia Natural en el portal SciELO, considerando el número de accesos entre Enero 2009 y Noviembre 2013 (fuente: SciELO).

Ranking of the 20 most consulted articles of Revista Chilena de Historia Natural in the SciELO portal, considering the number of accesses between January 2009 and November 2013 (source: SciELO).

\begin{tabular}{|c|c|c|c|}
\hline Ranking & Autor(es) & Título & $\begin{array}{l}\text { Vol. }\left(\mathrm{N}^{\circ}\right) \\
\text { año }\end{array}$ \\
\hline 1 & $\begin{array}{l}\text { LAUENROTH WK, EPSTEIN } \\
\text { HE, PARUELO JM et al. }\end{array}$ & $\begin{array}{l}\text { Potenciales efectos del cambio climático en zonas } \\
\text { templadas de América del Norte y del Sur }\end{array}$ & 77 (3) 2004 \\
\hline 2 & MUÑOZ-PEDREROS A & $\begin{array}{l}\text { La evaluación del paisaje: una herramienta de gestión } \\
\text { ambiental }\end{array}$ & 77 (1) 2004 \\
\hline 3 & $\begin{array}{l}\text { CARREÓN-PALAU A, URIA- } \\
\text { GALICIA E, ESPINOSA- } \\
\text { CHÁVEZ F }\end{array}$ & $\begin{array}{l}\text { Desarrollo morfológico e histológico del sistema } \\
\text { reproductor de Pomacea patula catemacensis (Baker } \\
\text { 1922) (Mollusca, Caenogastropoda: Ampullariidae) }\end{array}$ & 76(4) 2003 \\
\hline 4 & $\begin{array}{l}\text { FIGUEROA R, VALDOVINOS } \\
\text { C, ARAYA E et al. }\end{array}$ & $\begin{array}{l}\text { Macroinvertebrados bentónicos como indicadores de } \\
\text { calidad de agua de ríos del sur de Chile }\end{array}$ & $76(2) 2003$ \\
\hline 5 & $\begin{array}{l}\text { IRIARTE AJ, LOBOS GA, } \\
\text { JAKSIC FM }\end{array}$ & $\begin{array}{l}\text { Especies de vertebrados invasores en Chile y su control } \\
\text { y monitoreo por agencias gubernamentales }\end{array}$ & 78(1) 2005 \\
\hline 6 & $\begin{array}{l}\text { RIQUELME CE, AVENDAÑO- } \\
\text { HERRERA RE }\end{array}$ & $\begin{array}{l}\text { Interacción bacteria-microalga en el ambiente marino y } \\
\text { uso potencial en acuicultura }\end{array}$ & 76(4) 2003 \\
\hline 7 & $\begin{array}{l}\text { HALPIN PM, STRUB PT, } \\
\text { PETERSON, WT et al. }\end{array}$ & $\begin{array}{c}\text { Una visión general de las interacciones entre } \\
\text { la oceanografía, los ecosistemas marinos y las } \\
\text { disrupciones climáticas y antrópicas en los márgenes } \\
\text { este del Océano Pacífico }\end{array}$ & $77(3) 2004$ \\
\hline 8 & $\begin{array}{l}\text { CAVIERES LA, PEÑALOZA A, } \\
\text { KALIN-ARROYO M }\end{array}$ & $\begin{array}{c}\text { Pisos altitudinales de vegetación en los Andes de Chile } \\
\text { central }\left(33^{\circ} \mathrm{S}\right)\end{array}$ & $73(2) 2000$ \\
\hline 9 & $\begin{array}{l}\text { PÉREZ JE, MUÑOZ C, } \\
\text { HUAQUIN L, NIRCHIO M }\end{array}$ & $\begin{array}{l}\text { Riesgos de la introducción de tilapias (Oreochromis sp.) } \\
\text { (Perciformes: Cichlidae) en ecosistemas acuáticos de } \\
\text { Chile }\end{array}$ & 77 (1) 2004 \\
\hline 10 & $\begin{array}{l}\text { SILVA H, ACEVEDO E, SILVA } \\
\text { P }\end{array}$ & $\begin{array}{l}\text { Anatomía del tejido fotosintético de diez taxa de Opuntia } \\
\text { establecidos en el secano árido mediterráneo de Chile }\end{array}$ & $74(2) 2001$ \\
\hline
\end{tabular}




\begin{tabular}{|c|c|c|c|}
\hline Ranking & Autor(es) & Título & $\begin{array}{l}\text { Vol. }\left(\mathrm{N}^{\circ}\right) \\
\text { año }\end{array}$ \\
\hline 11 & $\begin{array}{l}\text { MARÍN JC, ZAPATA B, } \\
\text { GONZÁLEZ BA et al. }\end{array}$ & $\begin{array}{l}\text { Sistemática, taxonomía y domesticación de alpacas y } \\
\text { llamas: nueva evidencia cromosómica y molecular }\end{array}$ & $80(2) 2007$ \\
\hline 12 & $\begin{array}{l}\text { DEMERGASSO C, CHONG G, } \\
\text { GALLEGUILLOS P et al. }\end{array}$ & $\begin{array}{l}\text { Tapetes microbianos del Salar de Llamará, norte de } \\
\text { Chile }\end{array}$ & $76(3) 2003$ \\
\hline 13 & TORREJÓN F, CISTERNAS M & $\begin{array}{l}\text { Alteraciones del paisaje ecológico araucano por la } \\
\text { asimilación mapuche de la agroganadería hispano- } \\
\text { mediterránea (siglos XVI y XVII) }\end{array}$ & $75(4) 2002$ \\
\hline 14 & CAMUS PA & Biogeografía marina de Chile continental & $74(3) 2001$ \\
\hline 15 & $\begin{array}{l}\text { CECCHI MC, GUERRERO- } \\
\text { BOSAGNA C, MPODOZIS J }\end{array}$ & $\mathrm{El}$ ¿delito? de Aristóteles & $74(3) 2001$ \\
\hline 16 & CAMOUSSEIGHT A & $\begin{array}{l}\text { El aporte científico de Carl Linné a } 300 \text { años de su } \\
\text { nacimiento }\end{array}$ & $80(3) 2007$ \\
\hline 17 & DELGADO L, SEREY I & $\begin{array}{l}\text { Distribución del cobre en ecosistemas forestales de tipo } \\
\text { mediterráneo }\end{array}$ & $75(3) 2002$ \\
\hline 18 & $\begin{array}{l}\text { AIZEN MA, VÁZQUEZ DP, } \\
\text { SMITH-RAMÍREZ C }\end{array}$ & $\begin{array}{c}\text { Historia natural y conservación de los mutualismos } \\
\text { planta-animal del bosque templado de Sudamérica } \\
\text { austral }\end{array}$ & 75(1) 2002 \\
\hline 19 & MORRONE JJ & Hacia una biogeografía evolutiva & $80(4) 2007$ \\
\hline 20 & $\begin{array}{l}\text { PELLET PF, UGARTE E, } \\
\text { OSORIO E et al. }\end{array}$ & $\begin{array}{c}\text { Clave de taxones superiores de gorgojos sudamericanos } \\
\text { basada en caracteres de los adultos (Coleoptera, } \\
\text { Curculionoidea) }\end{array}$ & 78(1) 2005 \\
\hline
\end{tabular}

de consultas vía docencia no ha sido evaluada). En este sentido el alcance va aún más allá, y recientemente como parte del comité editorial de una revista científica para jóvenes de 14 a 22 años, principalmente estudiantes secundarios, pude constatar -con cierta sorpresa- que sus autores leen y citan regularmente a RCHN en sus artículos (véase; http://revista. cienciajoven.cl/).

\section{El rol de RCHN más allá de ISI}

¿Es "útil” RCHN fuera del ámbito estricto de la ciencia? Los párrafos anteriores parecen responder afirmativamente esta pregunta, y es muy satisfactorio comprobar que el impacto de RCHN no se restringe a la comunidad científica.

En una editorial de 1990, Ernst Hajek planteaba una visión crítica -quizá más pertinente hoy que cuando fue publicada- sobre los criterios usados para medir el valor de la ciencia, frente la creciente popularidad y uso de las mediciones de impacto, y se preguntaba sobre la utilidad real de los trabajos de RCHN en el manejo de recursos o la gestión de bienes naturales. En ella, Hajek llamaba la atención sobre cómo los evaluadores y medidores de ciencia -y los sistemas de evaluación de proyectos o de académicos- persisten en "mirar más hacia fuera que hacia dentro de un país", y cómo la presión evaluativa que envuelve a los académicos "los obliga siempre a estar más pendientes del puntaje que les dará una publicación”. En este contexto, sugería además que "Sería interesante poder hacer consultas colectivas respecto de cuál es efectivamente el aprovechamiento que se puede hacer de los trabajos básicos del área naturalista, en términos de la ayuda que implica su existencia para lograr el desarrollo de nuestros países y lograr un uso sostenible en el tiempo de todos sus bienes naturales y, con ello, proveer una adecuada calidad de vida a sus habitantes" (Hajek 1990).

Creo que la última sección de este artículo contesta al menos en parte, y en forma positiva, algunas de las inquietudes formuladas por Hajek, y espero que la revista pueda seguir 
cumpliendo funciones relevantes en la educación y la gestión ambiental, sin olvidar sus vínculos y responsabilidad con la comunidad científica regional que la ha sostenido hasta ahora.

AGRADECIMIENTOS: Mi nexo de 19 años con RCHN ha sido una oportunidad de aprender en muchos sentidos, y siempre he disfrutado aprender así que debo agradecer a varios. A todos los Editores de la revista en su era moderna: Ernst Hajek, con quien nunca trabajé pero tuve la suerte de conocerlo y de apreciar su notable visión y sensatez; Fabián Jaksic, que me enroló como Editor de Producción y me dejó hacer y deshacer, en el buen sentido; Patricio Ojeda, que me "ascendió" a Editor Asociado con lo que pude conocer otras facetas editoriales; y Luis Ebensperger que me permitió continuar y luego me sugirió como su reemplazo (aunque pensándolo bien quizá debería culparlo por eso). A todos los Editores Asociados con quienes tuve el gusto de compartir, y que en muchos casos desplegaban niveles de rigurosidad y calidad admirables. A los tres presidentes de la Sociedad de Biología de Chile que me confiaron la revista: Tulio Núñez, P. Ojeda y Rosalba Lagos. A mis Editores de Producción: Carlos Zamora-Manzur, fundamental en todos las acciones electrónicas que implementamos o intentamos en la revista (incluyendo sobrellevar un terremoto $8.8^{\circ}$ Richter), y Paulina A. Arancibia que aportó un impulso de orden y eficiencia. Finalmente al Fondo de Publicación de Revistas Científicas de CONICYT, que con los proyectos FP 11032 y FP 0949 nos permitió efectuar mejoras significativas en la revista.

\section{LITERATURA CITADA}

BAHAMONDE N (1983) Don Carlos Emilio Porter Mosso. Sabio naturalista chileno. Revista Chilena de Historia Natural 56: 7-9.

CAMUS PA (1995) El ámbito temático de la Revista Chilena de Historia Natural: un análisis de las principales tendencias observadas entre 1983 y 1995. Revista Chilena de Historia Natural 68: 409-417.

COURARD H (1986) Estudios del campo científico VIII. Notas sobre el desarrollo de la biología en Chile 1750-1950. Programa FLACSO, Santiago, Chile. URL: http://cronopio.flacso.cl/fondo/pub/ publicos/1986/DT/000834.pdf

ETCHEVERRY M (1985) Datos sobre la Revista Chilena de Historia Natural. Revista Chilena de Historia Natural 58: 83-92.

ETCHEVERRY M (1988) Datos biográficos sobre don Carlos Emilio Porter Mossó (1867-1942). Revista Chilena de Historia Natural 61: 127-128.

GIGLIA E (2010) The impact factor of open access journals: data and trends. $14^{\text {th }}$ International Conference on Electronic Publishing, Helsinki. URL: http://elpub.scix.net/data/works/att/102_ elpub2010.content.pdf

HAJEK ER (1990) Investigación básica en las ciencias naturales: la necesidad de medir su impacto en, y para, los países en desarrollo. Revista Chilena de Historia Natural 63: 145-148.

JAKSIC FM (1997) The first hundred years of the Revista Chilena de Historia Natural. Revista Chilena de Historia Natural 70: 5-8.

JAKSIC FM, P CAMUS \& SA CASTRO (2012) Ecología y ciencias naturales. Historia del conocimiento del patrimonio biológico de Chile. Dirección de Bibliotecas, Archivos y Museos, Santiago, Chile.

LUNA-MORALES ME \& F COLLAZO-REYES (2007) Análisis histórico bibliométrico de las revistas latinoamericanas y caribeñas en los índices de la ciencia internacional: 1961-2005. Revista Española de Documentación Científica 30: doi:10.3989/ redc.2007.v30.i4.403 